\title{
48. PROGRESS REPORT ON THE CLEVELAND-CHILE SURVEY FOR SOUTHERN OB STARS
}

\author{
C. B. STEPHENSON and N. SANDULEAK \\ Warner and Swasey Observatory, Cleveland, Ohio, U.S.A.
}

From some standpoints it should seem surprising that not all of the OB stars of our Galaxy that can be identified to a feasible limiting magnitude (say 12-13) by conventional objective prism techniques have yet been so identified, but this is in fact the state of our published data. Some people here will recall that such a systematic survey for the northern Milky Way was carried out several years ago jointly by the Hamburg and Warner and Swasey Observatories. Now at the Warner and Swasey Observatory we are extending this northern survey into the southern Milky Way that could not be reached from the north, and we are making this extension as homogeneous as possible with respect to the northern survey.

We expect our goal of homogeneity to be achieved because of two factors: Stephenson was extensively involved in the northern survey, and the southern extension is being accomplished with equipment nearly identical to that used in Cleveland, viz. the Michigan Schmidt telescope (now at Cerro Tololo) and the same objective prism as used at Cleveland. The chief difference between the new and old plate material is that the new plates are of superior quality to the Cleveland ones, mostly because the Michigan Schmidt is optically superior to the Warner and Swasey one and partly because of the good seeing at Cerro Tololo; however, the southernmost Chile plates are quite variable in limiting magnitude, for reasons partly known and partly unknown.

We expect to publish our catalogue and identification charts in 1970, and a major purpose of our presenting this report is to solicit opinions as to the most useful publishing format. We will probably adopt at least the following changes with respect either to the northern catalogues or to common southern-hemisphere practice: (1) We shall arrange the stars by right ascension throughout, instead of by declination zones. (2) We shall give CD numbers in preference to CPD numbers, even though most southern workers for some reason are doing the opposite. Our reason for this choice is that CD charts exist for the whole southern sky, while the same is not true of the CPD. (3) We are considering including an index that will cross-reference our star numbers against those of previous surveys. (4) The charts will be prepared from direct plates, probably of the scale of the Lick Sky Atlas, rather than from objective prism plates as was done for the Cleveland zones (no charts were ever published for the Hamburg zones).

Finally we should mention that we are receiving numerous requests for pre-publication data from this survey. Because of this we should point out that such data exist at present only in the form of handwritten material, which is not yet computer- 
compatible and is thus very time-consuming to prepare for transmission to a potential user, and this will remain true almost up to the time when we submit our manuscripts for publication. For this reason we would like to apologise for our having to decline requests for such material for about another year, unless the need for it should be truly exceptional. 